\title{
The low-mass dispersed population around the Lupus clouds ${ }^{\star}$
}

\author{
F. Comerón ${ }^{1, \star \star}$, L. Spezzi ${ }^{1}$, B. López Martí ${ }^{2}$, and B. Merín ${ }^{3}$ \\ 1 ESO, Karl-Schwarzschild-Strasse 2, 85748 Garching bei München, Germany \\ e-mail: fcomeron@eso.org \\ 2 Centro de Astrobiología (INTA-CSIC), PO Box 78, 28691 Villanueva de la Cañada, Madrid, Spain \\ 3 Herschel Science Centre, European Space Astronomy Centre (ESA), SRE-OAH, PO Box 78, 28691 Villanueva de la Cañada, \\ Madrid, Spain
}

Received 3 August 2012 / Accepted 29 March 2013

\section{ABSTRACT}

\begin{abstract}
Context. Many star forming regions are known to have associated dispersed populations, whose members are located away from known current star forming sites. Their origin is unclear, and any identification of the members through relatively short-lived signatures of youth can miss them.

Aims. We aim at confirming membership of a sample of cool stars identified in a previous work in the Lupus 1,3 , and 4 clouds as candidate members. Most of them do not display near- or mid-infrared excess or any other easily recognizable signatures of youth. Methods. We use low-resolution spectroscopy in the red part of the spectrum, including the $\mathrm{H} \alpha$ region, to accurately determine spectral types and probe surface gravity-sensitive features that provide reliable criteria for distinguishing cool giant stars, young stellar objects, and evolved dwarf stars.

Results. Most of the candidate members of a possible dispersed population around Lupus 1 are found to be background $\mathrm{K}$ or early $\mathrm{M}$ giants. However, about half of the observed members of Lupus 3 are confirmed as young objects, including both low-mass stars and brown dwarfs. The distributed population is compared to the sample of lightly obscured members projected closer to the densest parts of the Lupus 3 star forming cloud, and the estimated ages of the members of both sets are found to be consistent with a single distribution. However, we find statistical indications (although at a low significance level) of a decrease in the frequency of infrared excesses in the distributed population. Some nongiant members are also identified with gravity-sensitive features typical of more evolved stars, and we argue that these may belong to an older population associated with the Gould Belt, similar to what is observed in the direction of other nearby star forming regions. We also confirm two additional, very low-mass members of Lupus 4.

Conclusions. Although some of its members have already been known previous to this work, our results emphasize the richness of the low-mass distributed population around Lupus 3 and the existence of much less numerous dispersed populations around Lupus 1 and Lupus 4. The apparent spatial segregation as a function of the abundance of circumstellar material favors dynamical ejection from the main star forming cloud as the mechanism that gives rise to the dispersed population.
\end{abstract}

Key words. stars: low-mass - brown dwarfs - stars: formation - stars: pre-main sequence - ISM: individual objects: Lupus ISM: clouds

\section{Introduction}

Newly formed stars in their birth sites display a wide variety of the signposts of youth. Many of them relate to the presence of massive circumstellar disks and envelopes that reprocess at infrared and millimeter wavelengths the light emitted by the central object, provide a reservoir of material for accretion onto its surface, and feed magnetically-driven outflows. While the presence of strong accretion, outflow activity, and substantial circumstellar disks is relatively short-lived (e.g. Ray 2007, Evans et al. 2009, Luhman et al. 2010), some of the observational manifestations of youth, such as residual accretion or the emission at high energies due to vigorous chromospheric activity, can last for tens of millions of years (Feigelson \& Montmerle 1999), providing observational diagnostics for detecting young stellar populations. Ultimately, even in the absence of such manifestations of youth, pre-main sequence stars still reveal their nature through more subtle photospheric indicators of their early phases of contraction, such as gravity-sensitive spectroscopic features or the

$\star$ Based on observations collected with the Very Large Telescope (VLT) at the European Southern Observatory, Paranal, Chile, under observing program 086.C-0546(A).

$\star \star$ Visiting astronomer at the Vatican Observatory. incomplete depletion of lithium due to a still insufficient central temperature. Observations of young stellar objects lacking the most obvious signposts of youth are very important for obtaining complete samples of the stellar populations of young aggregates, for tracing their star formation history back in time, for estimating the evolutionary timescale of circumstellar matter, or for assessing the importance of events that may result in its early loss.

In Comerón et al. (2009, hereafter CSL) we published the results of an imaging survey of three star forming clouds in Lupus and their surroundings in the $R_{\mathrm{C}}, I_{\mathrm{C}}$, and $z$ bands that, combined with 2 MASS $J H K_{\mathrm{S}}$ photometry (Skrutskie et al. 2006), provided a sampling of the spectral energy distribution of all the sources in the field between 0.6 and $2.2 \mu \mathrm{m}$. The main purpose of those observations was to complete in the visible the sampling of the infrared spectral energy distribution carried out by the Spitzer Space Telescope in the Lupus clouds (Merín et al. 2008) as part of its legacy program "From molecular cores to star-forming disks" (Evans et al. 2003). The 0.6-2.2 $\mu \mathrm{m}$ range contains the peak of the photospheric emission of the cold photospheres of very low mass stars and massive brown dwarfs, making the disentanglement of an intrinsically red spectral energy distribution and the reddening caused by foreground dust robust. In this way 
we could identify cold stars with $T_{\text {eff }} \sim 3500-4000 \mathrm{~K}$ and below, including cold, low-mass young stellar objects. Furthermore, we made use of the fact that the distribution of apparent fluxes of cold objects at the galactic latitudes of the Lupus clouds is split into two components, a faint one populated by field dwarfs and a brighter one populated by background giants, leaving a gap between both populations that is statistically expected to be almost depopulated. The low-mass stars and brown dwarfs of young aggregates lying between $\sim 100 \mathrm{pc}$ and $\sim 300 \mathrm{pc}$ from the Sun and having ages below $\sim 10 \mathrm{Myr}$ fall precisely in this gap, producing a distinctive signature in the histogram of dereddened apparent magnitudes of objects of a given temperature.

The Lupus clouds that we targeted are placed at 150-200 pc from us (Comerón 2008) and the members of their confirmed populations have ages of a few Myr or less. This makes our observations of the Lupus clouds very well suited for the identification of candidate members on the sole basis of their broad-band colors and their fluxes.

The analysis of the imaging of Lupus presented in CSL clearly shows the statistical signature of many previously unrecognized cold members of the Lupus 3 cloud, in addition to the numerous members already known from previous studies. The new members are mainly located in a distributed population, projected at large angular distances from the main dark clouds where the most recent star formation is taking place at present. Indications were also discussed on a similar population in Lupus 1. However, the results were more uncertain in that case due to them being based on stars of higher temperature, where the wavelength range probed by our broad-band photometry starts probing the Rayleigh-Jeans tail of the spectral energy distribution. Finally, no clear signature of a previously unknown population was found in Lupus 4 , although a few candidate new members were identified. A common characteristic of the vast majority of new candidates is that they appear projected outside the boundaries of the dark clouds that contain most of the previously known members, suggesting the presence of extended haloes of young stars around the currently observed clouds. However, the information that one may obtain from broad band photometry alone yields little light on the nature of this distributed population.

In this paper we present red visible spectroscopy of most of the candidate new members of the Lupus 1, 3, and 4 clouds identified by CSL. The spectra allow us to obtain accurate spectral classifications and thus refine the estimates of temperature, luminosity, extinction, mass and age previously available from the photometry. The new results presented here essentially confirm our previous findings on Lupus 3, but show that the new population of Lupus 1 is much less important in number than derived in CSL. We discuss the implications of our results, combined with previously existing observations in the infrared, on the nature of the distributed population of Lupus 3.

\section{Observations}

The long-slit spectroscopy presented in this paper was obtained with the FORS2 camera and spectrograph at the Very Large Telescope (VLT) in service mode, over several nights distributed between 1 February and 31 March 2011. A grism covering the spectral region between $6000 \AA$ and $11000 \AA$ was used in combination with a slit $1^{\prime \prime} 0$ wide, providing a resolution $\lambda / \Delta \lambda=660$. Exposure times were adjusted according to the $R_{\mathrm{C}}$ magnitude of each star, and ranged from 2 min for the brightest sources to $126 \mathrm{~min}$ for the faintest. The exposures were split in blocks no more than $60 \mathrm{~min}$ long in duration, including the overheads due to telescope presetting, detector readout, and offsets between exposures. When two or more blocks were needed due to the faintness of the star, they were usually executed on different nights. Standard calibrations provided by the instrument calibration plan were used. These included bias and flat field frames, arc lamp exposures for wavelength calibration, and at least one spectrophotometric standard obtained each night. Data reduction, extraction of one-dimensional spectra, and wavelength and relative flux calibration was carried out using standard $\mathrm{IRAF}^{1}$ tasks.

The whole list of candidate new members of Lupus 1, 3, and 4 presented by CSL was submitted as targets of our observations, with no predetermined priority of execution. The observatory then selected targets at random for execution when the conditions were suitable. At the end of the observing period, 91 targets of the 143 submitted had been observed (40 out of 65 in Lupus 1, 46 out of 72 in Lupus 3, and 5 out of 6 in Lupus 4), thus providing a significant sampling of the new candidates population of each cloud.

\section{Analysis}

The spectral region covered by our observations is well suited for the classification of stars in the range of temperatures expected from the spectral energy distribution fits carried out by CSL $\left(T_{\text {eff }} \lesssim 3700 \mathrm{~K}\right.$ in Lupus $1, T_{\text {eff }} \lesssim 3400 \mathrm{~K}$ in Lupus 3 and 4), providing diagnostic features of temperature and surface gravity as well as accretion/outflow diagnostic lines near $\mathrm{H} \alpha$. It also includes the LiI line at $6708 \AA$, but unfortunately the combination of resolution and signal-to-noise ratio of our spectra provide only upper limits of no practical significance given the typical equivalent width of this line as measured in similar objects (e.g. Neuhäuser \& Comerón 1999). The CaII triplet absorption lines at $8498 \AA, 8542 \AA$ and $8662 \AA$ are very prominent in $\mathrm{K}$ and early-M giants (Kirkpatrick et al. 1991), thus providing a straightforward criterion for discriminating between background contaminants and candidate members of the star forming region. Clear differences between giants and nongiants exist also at later spectral types, such as the rounded top of the broad feature peaking near $7000 \AA$ or the flux drop redwards of $8200 \AA$, thus extending the discrimination criteria to the entire range of spectral types relevant to this study. The strong temperature dependency of the $\mathrm{TiO}$ and $\mathrm{VO}$ molecular bands in M stars allows us to carry out an accurate spectral typing of the coolest stars in our sample. At earlier types, the spectra have a much smoother appearance dominated at wavelengths shorter than $9000 \AA$ by the strong telluric $\mathrm{O}_{2}$ features near $6870 \AA$ and $7600 \AA$, with weak or no indications of the TiO bands that dominate M-type spectra, thus making the determination of the spectral subtype and the estimate of the temperature more uncertain.

Spectral types for the giant stars found in our sample and recognized through the gravity-sensitive features noted above have been assigned by comparison to the spectra of $\mathrm{K}$ giants presented by Kirkpatrick et al. (1991) for types earlier than M5, and by Fluks et al. (1994) for later types. The red spectral range covered in our study becomes unsuitable for the accurate classification of $\mathrm{K}$ giants, and we are unable to provide a spectral subtype classification for stars earlier than K5.

We have used the extensive atlas of spectra of very young low-mass stars and brown dwarfs in Chamaeleon I by Luhman (2007) for the classification of nongiant stars in

1 IRAF is distributed by NOAO, which is operated by the Association of Universities for Research in Astronomy, Inc., under contract to the National Science Foundation. 
our sample. The wavelength coverage and resolution used by Luhman (2007) are similar to those of our spectra, and we are able to determine spectral types to the nearest half subclass. In addition to the accurate determination of spectral types, our spectra provide us with diagnostics of surface gravity, most notably the NaI line doublet around $8190 \AA$, a prominent feature over the entire $M$ spectral range whose depth increases with increasing surface gravity. We have compared the depth of this feature in the spectra of our objects with the NextGen synthetic spectra of Allard et al. (2000), which are appropriate for the temperature range of interest $\left(T_{\text {eff }}>2700 \mathrm{~K}\right)$, smoothed to the resolution of our observations. The surface gravity is estimated by comparing the NaI feature in our spectra with that of the sequence of synthetic spectra of the same temperature and varying $\log g$, and we adopt as the value of $\log g$ of our objects that of the closest matching synthetic spectra. The spacing of $\log g$ values in the grid of synthetic spectra corresponds well with our ability to discern variations in the depth of the NaI feature, thus yielding an accuracy of approximately $0.5 \mathrm{dex}$ in our estimate of $\log g$. Evolutionary tracks for low-mass stars and massive brown dwarfs (Baraffe et al. 1998) indicate that values of $\log g=3.5,4.0$, and 4.5 are reached at ages of 1, 5, and $25 \mathrm{Myr}$ approximately, reaching $\log g=5.2$ for cool dwarfs at the bottom of the main sequence (Gorlova et al. 2003). Therefore, the resolution achieved with our comparison is sufficient to carry out a rough dating of our objects within the age range expected in the targeted regions. For the stars whose $\mathrm{NaI}$ feature indicates a surface gravity comparable to those of field $\mathrm{M}$ dwarfs, spectral types have been determined by comparison to the atlas of Kirkpatrick et al. (1991).

Almost all the stars in our sample can be classified as having $\mathrm{K}$ or $\mathrm{M}$ spectral types. The only exception is J160937.4-391044, whose prominent hydrogen lines classify it as a A-type star. This star is excluded from the ensuing discussions.

\section{Results}

\subsection{Background giants}

The CaII triplet absorption lines at $8498 \AA$, $8542 \AA$ and $8662 \AA$ are very strong in nearly all the objects with spectral type earlier than M, with the single exception of the young X-ray emitting star J160836.2-392302 (Krautter et al. 1997). This clearly indicates that these objects are red giants unrelated to the Lupus clouds. Some additional giants are identified among the early $\mathbf{M}$ stars, also thanks to their CaII triplet absorption, and among the late ones using other characteristic spectral features described in Sect. 3. Figure 1 displays the spectra of all the giant stars in our sample with spectral types later than M0, and the complete list of giant stars in the sample is given in Table 1.

The large number of giants contained in our sample (59 out of 91 objects observed) may be explained as a consequence of the rapid increase in the number of these stars toward earlier spectral types predicted by galactic star count models, as already pointed out by CSL. Furthermore, the CSL estimate of $T_{\text {eff }}$ strongly relies on the sampling of the peak of the spectral energy distribution. As the peak shifts toward shorter wavelengths outside the range covered by broad band photometry with increasing $T_{\text {eff }}$, temperature and extinction effects become harder to disentangle at the time of fitting both simultaneously as done by CSL, thus making the temperature estimates presented there progressively inaccurate. As expected, the number of giant contaminants is greater in the direction of Lupus 1 due to the limit

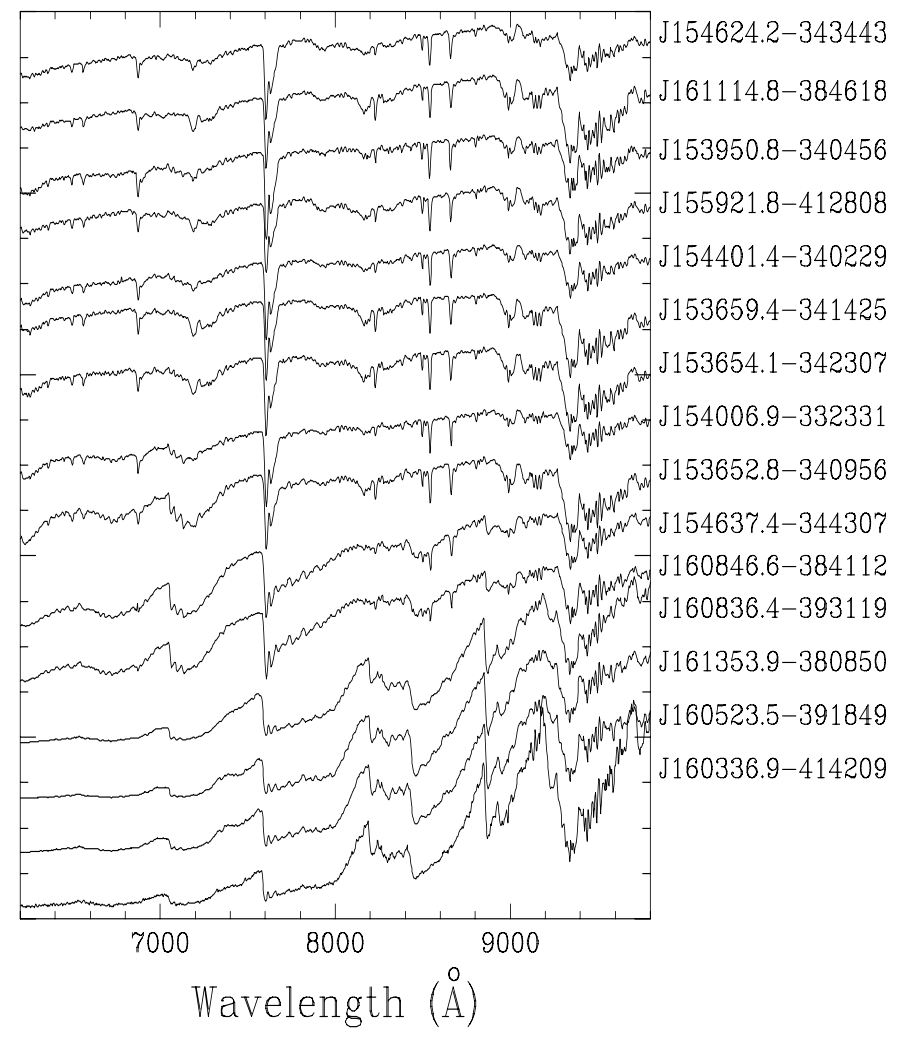

Fig. 1. Sample spectra of candidate members of the Lupus star forming region whose membership is ruled out by our new observations. The spectra presented here correspond to the coolest non-members having M0 or later spectral types. The prominent CaII triplet absorption lines at $8498 \AA, 8542 \AA$ and $8662 \AA$ are clear indicators of very low surface gravities indicating that the stars are actually giants. Giant stars in our sample earlier than M0, not shown here, are also identified through strong CaII absorption. The CaII lines in absorption disappear at mid-M types, but other surface gravity indicators discussed in the text clearly show that the bottom four stars are giants as well.

of $T_{\text {eff }}<3700 \mathrm{~K}$ chosen by CSL for the selection of candidates. Due to their lower galactic latitude and the subsequent greater number of background contaminants, this limit was reduced to $T_{\text {eff }}<3400 \mathrm{~K}$ in Lupus 3 and 4 . The vast majority of background giants included in our sample have spectral types between K5III and M2III, thus covering the $3700 \lesssim T_{\text {eff }} \lesssim 4000$ range (Decin et al. 2003), not far from the selection cutoff.

The abundance of giants in our sample largely contributes to explaining the kinematic patterns in the region reported by López Martí et al. (2011). The sample studied by those authors includes all confirmed and candidate members of the Lupus clouds for which astrometric information is available. They find two distinct kinematical groups, one containing most of the confirmed Lupus members and another displaying smaller proper motions that they tentatively ascribe to a background giant population. By comparing to the list of members of each group, 51 out of the 52 stars of the second group for which we have obtained spectroscopy are indeed confirmed as giants. The only nongiant in the group is J161033.2-383023, a likely member of the Lupus 3 population. The results are more mixed in the first group, for which we obtained spectroscopy of 15 members, of which 8 are confirmed as nongiants. However, the fraction of Lupus members in this group is much higher when including known members of the young population already confirmed by 
Table 1. Giant stars.

\begin{tabular}{|c|c|c|c|}
\hline Star & Type & Star & Type \\
\hline Lupus 1 & & Lupus 3 & \\
\hline J153652.8-340956 & M2 & $\mathrm{J} 160509.3-391203$ & K5 \\
\hline J153654.1-342307 & K7 & J160523.5-391849 & M7.5 \\
\hline J153659.4-341425 & M0 & J160624.4-392158 & K5 \\
\hline $\mathrm{J} 153736.0-345902$ & K7 & $\mathrm{J} 160727.1-391601$ & $<\mathrm{K} 5$ \\
\hline $\mathrm{J} 153742.9-332034$ & K5 & J160735.3-392507 & K5 \\
\hline $\mathrm{J} 153745.7-342920$ & K7 & $\mathrm{J} 160747.4-392606$ & K5 \\
\hline J153750.9-332722 & K5 & J160836.4-393119 & M6.5 \\
\hline J153753.2-343256 & K7 & $\mathrm{J} 160844.3-374443$ & K5 \\
\hline J153756.8-342117 & K7 & $\mathrm{J} 160846.4-393347$ & $<\mathrm{K} 5$ \\
\hline J153805.7-341535 & K5 & J160846.6-384112 & M4.5 \\
\hline J153808.7-343557 & $<\mathrm{K} 5$ & J160855.6-392316 & $<\mathrm{K} 5$ \\
\hline $\mathrm{J} 153820.2-350215$ & K5 & $\mathrm{J} 160857.3-392849$ & $<\mathrm{K} 5$ \\
\hline $\mathrm{J} 153821.4-341519$ & K7 & $\mathrm{J} 160903.7-385610$ & K5 \\
\hline J153824.8-340629 & K5 & J160903.8-384126 & K5 \\
\hline J153831.6-331031 & K7 & J160917.6-392537 & K5 \\
\hline $\mathrm{J} 153859.5-343458$ & K5 & J160939.5-384431 & $<\mathrm{K} 5$ \\
\hline J153900.3-345534 & K5 & $\mathrm{J} 161001.1-384315$ & K5 \\
\hline J153902.1-342040 & $<\mathrm{K} 5$ & $\mathrm{~J} 161013.5-384208$ & K7 \\
\hline $\mathrm{J} 153905.2-341210$ & K5 & $\mathrm{J} 161036.7-380927$ & K5 \\
\hline J153922.3-334909 & $<\mathrm{K} 5$ & $\mathrm{~J} 161114.8-384618$ & K7 \\
\hline J153929.0-340339 & K7 & $\mathrm{J} 161200.9-383625$ & $<\mathrm{K} 5$ \\
\hline $\mathrm{J} 153933.2-350151$ & K5 & $\mathrm{J} 161229.0-383420$ & K5 \\
\hline J153940.8-333941 & K5 & $\mathrm{J} 161353.9-380850$ & M7.5 \\
\hline J153943.8-341506 & K5 & & \\
\hline $\mathrm{J} 153950.8-340456$ & M0 & Lupus 4 & \\
\hline J153955.6-341103 & $<\mathrm{K} 5$ & & \\
\hline $\mathrm{J} 154006.9-332331$ & M0 & J155921.8-412808 & K7 \\
\hline $\mathrm{J} 154009.4-342734$ & $<\mathrm{K} 5$ & J160336.9-414209 & M8 \\
\hline $\mathrm{J} 154013.7-340142$ & K5 & & \\
\hline J154344.5-335834 & K7 & & \\
\hline J154401.4-340229 & M0 & & \\
\hline $\mathrm{J} 154624.2-343443$ & M0 & & \\
\hline J154637.4-344307 & M4.5 & & \\
\hline
\end{tabular}

previous studies. The contamination of the population of each kinematic group by members of the other population can be explained by the scatter around the average proper motions of each group, which causes some overlap between both, as well as by the higher scatter in proper motion of the kinematic group dominated by giants. A large part of this scatter is caused by large proper motion errors in available astrometric catalogs. In a recent kinematical study on the young associations in the Chamaeleon sky area, López Martí et al. (in prep.) show that up to $10 \%$ of the candidate members of Chamaeleon I from the literature later identified as background contaminants through spectroscopy have proper motions overlapping with those of the group of spectroscopically confirmed members. Likewise, radial velocity information allows them to discard some candidate members from the ROSAT All-Sky Survey displaying similar proper motion as the young stars in the associations, and for which spectroscopy had been inconclusive about their origin.

\subsection{Cool young stellar objects and dwarfs}

Out of our 91 observed targets, 32 are spectroscopically confirmed as nongiants. Apart from the young K5 star J160836.2-392302, all the other nongiant targets of our sample have spectral types comprised between M2.5 and M7.5, thus spanning the range of low-mass stars and massive brown dwarfs.

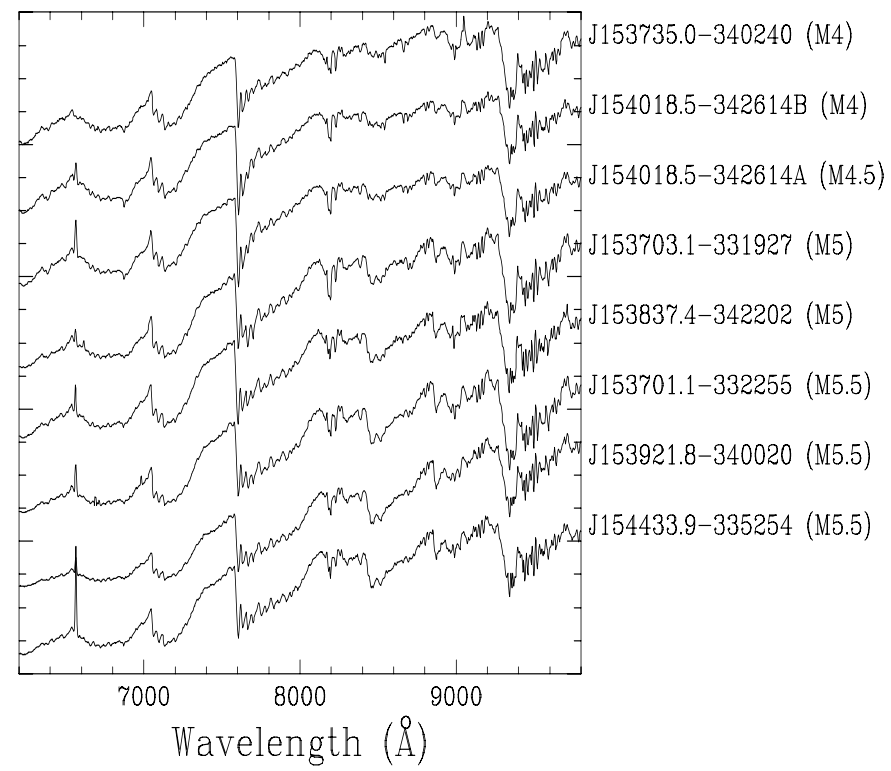

Fig. 2. Spectra of the nongiant stars observed in the proximities of the Lupus 1 cloud.

The spectra of the nongiants found in each of the three regions are displayed in Figs. 2-4. In addition to the range of spectral types covered by our objects, a detailed examination shows that the range of strengths of the NaI feature is also large. Our results also hint at differences between regions in this respect, as can be seen by comparing Figs. 2, 3, where NaI depths tend to be greater in the former.

\subsection{Intrinsic properties}

The results of fits to the spectral energy distributions of the nongiant stars and brown dwarfs in our sample are summarized in Table 2. The fitting procedure is an adaptation of the one described in Spezzi et al. (2007) and CSL. Unlike in those works, here we use only one synthetic spectrum to fit the photometry of each object. The synthetic spectrum is taken from the grid of Allard et al. (2000), using both the $T_{\text {eff }}$ and the $\log g$ derived from the actual object spectrum to make the choice. In this way, only the extinction and the luminosity are derived through a leastsquare fit. Like in CSL, the luminosity is derived by assuming a common distance of $150 \mathrm{pc}$ for all the objects seen in the direction of Lupus 1 and 4, and of 200 pc for those in the direction of Lupus 3, following the discussion in Comerón (2008). Ages and masses are derived using the Baraffe et al. (1998) evolutionary tracks for masses above the substellar limit $\left(0.075 M_{\odot}\right)$, complemented by those computed by Chabrier et al. (2000) for massive brown dwarfs with dusty atmospheres. No luminosities, masses or ages are provided for objects with $\log g \gtrsim 4.5$, as those are considered to be likely non-members foreground to the Lupus clouds as discussed in Sect. 5.5.

With the sole exception of J153735.0-340240, all the other nongiant objects of our sample display low to moderate $\mathrm{H} \alpha$ emission. Its measured equivalent width, $E W(\mathrm{H} \alpha)$, is also given in Table 2. For each object, we also computed the spectral index $n=d \log \lambda f_{\lambda} / d \log \lambda$, approximated by $n=$ $-3-0.4\left(m_{\lambda_{2}}-m_{\lambda_{1}}\right) / \log \left(\lambda_{2} / \lambda_{1}\right)$, where $\lambda_{1}$ and $\lambda_{2}$ are respectively the lower and upper limit of the range in which the spectral energy distribution slope is measured, and $m_{\lambda_{1}}, m_{\lambda_{2}}$ are the magnitudes at those wavelengths. These magnitudes are listed for 
F. Comerón et al.: The low-mass dispersed population around the Lupus clouds
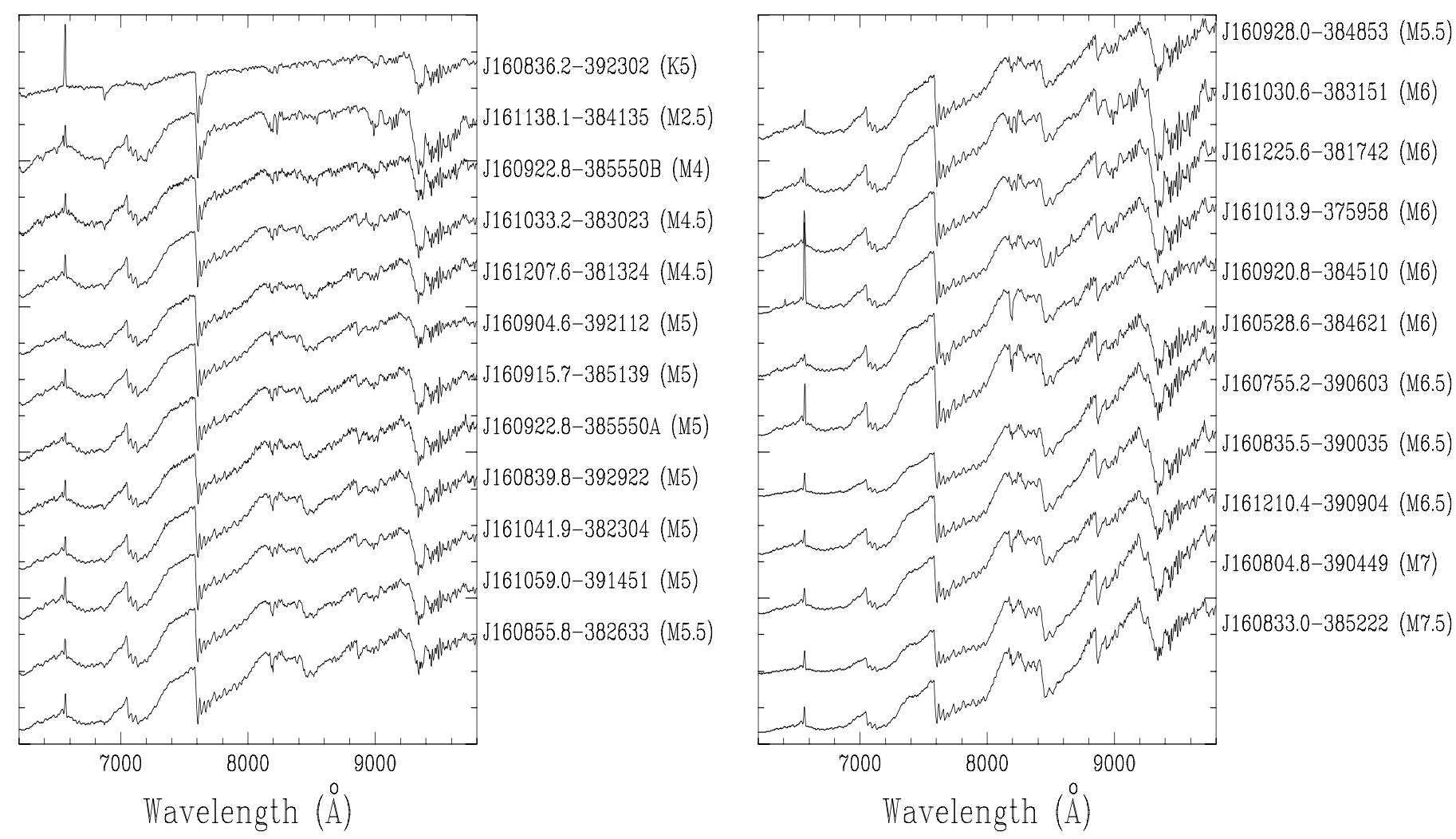

Fig. 3. Spectra of the nongiant stars observed in the proximities of the Lupus 3 cloud.

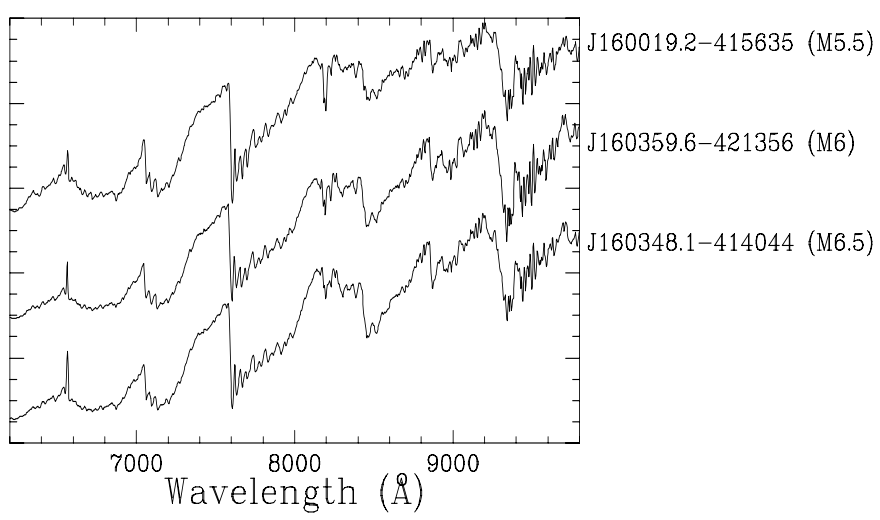

Fig. 4. Spectra of the nongiant stars observed in the proximities of the Lupus 4 cloud.

most objects in CSL based on the fluxes reported by Merín et al. (2008). Normally we use $\lambda_{1}=2.2 \mu \mathrm{m}$ and $\lambda_{2}=24 \mu \mathrm{m}$, but in the two cases in which the long wavelength measurement is missing because the star is too faint at the $24 \mu \mathrm{m}, \lambda_{2}=8 \mu \mathrm{m}$ has been used. In the cases for which no value of $n$ is given in Table 2 the object falls below the detection limits of Spitzer in all bands, and can thus be safely considered not to display any significant infrared excess up to at least $70 \mu \mathrm{m}$.

\section{Discussion}

The new information provided by the available spectroscopy allows us to reassess the reality of the cool stellar populations around Lupus 1 and 3 reported by CSL and to obtain additional clues about their possible origin. The $T_{\text {eff }}-L$ diagrams presented in Fig. 5, constructed from the data presented in Table 2, provide additional hints on physical membership to each of the regions.

\subsection{Lupus 1}

Our earlier claim of a numerous population of new members around Lupus 1, based on the statistics of stars fitted by a cool photosphere, is largely disproved by the available spectroscopy, which shows that over $80 \%$ of the selected candidates observed are actually background giants. However, a few nongiant candidates are retained. Figure 2 and Table 2 show that the $\log g$ values of those candidates are generally high, and more than half of them have $\log g$ in the 4.5-5.0 range, suggesting ages well above 10 Myr. This includes J154018.5-342614B, a serendipitously discovered close companion to J154018.5-342614 that happened to fall within the slit of the spectrograph at the time when the observations were carried out. Both members of the pair have very similar spectral types, surface gravities and $\mathrm{H} \alpha$ emission. The surface gravities of the remaining three objects suggest ages perhaps in the 5-10 Myr range. Based on the spectral types, all the candidates in Lupus 1 appear to be low-mass stars rather than brown dwarfs.

The comparison between the positions of the stars to which we assign $\log g=4.0$ in the $T_{\text {eff }}-L$ diagram of Lupus 1 (left panel of Fig. 5) and the isochrones at the adopted distance to that cloud shows a good agreement between the ages inferred from the derived luminosities and the range expected from their $\log g$. One of those stars, J154433.9-335254, displays moderate $\mathrm{H} \alpha$ emission, whereas the equivalent widths of the other stars are typical of weak-line T Tauri stars or of field dMe stars. The expectation that the objects with higher values of $\log g$ should be more evolved and thus less luminous is not confirmed by their 
Table 2. Parameters of nongiant cool stars.

\begin{tabular}{|c|c|c|c|c|c|c|c|c|c|}
\hline Object & $\begin{array}{l}T_{\text {eff }} \\
(\mathrm{K})\end{array}$ & $\begin{array}{l}\text { Sp. } \\
\text { type }\end{array}$ & $\begin{array}{c}\log g \\
\left(\mathrm{~cm} \mathrm{~s}^{-2}\right) \\
\end{array}$ & $\begin{array}{c}A_{V} \\
(\mathrm{mag})\end{array}$ & $\begin{array}{c}\log L^{a} \\
\left(L_{\odot}\right) \\
\end{array}$ & $\begin{array}{c}M^{a} \\
\left(M_{\odot}\right)\end{array}$ & $\begin{array}{l}\text { Age }^{a} \\
(\mathrm{Myr})\end{array}$ & $\begin{array}{c}E W(\mathrm{H} \alpha) \\
(\AA)\end{array}$ & $n$ \\
\hline \multicolumn{10}{|l|}{ Lupus 1} \\
\hline J153701.1-332255 & 3050 & M5.5 & $4.5^{b}$ & & & & & 12.4 & - \\
\hline J153703.1-331927 & 3100 & M5 & $5.0^{b}$ & & & & & 4.4 & - \\
\hline J153735.0-340240 & 3250 & M4 & $4.5^{b}$ & & & & & 0.0 & - \\
\hline J153837.4-342202 & 3100 & M5 & 4.0 & 1.2 & -1.74 & 0.15 & 8.0 & 7.8 & - \\
\hline J153921.8-340020 & 3050 & M5.5 & 4.0 & 3.4 & -1.68 & 0.10 & 7.1 & 9.5 & - \\
\hline J154018.5-342614A & 3200 & M4.5 & $4.5^{b}$ & & & & & 7.8 & -2.8 \\
\hline J154018.5-342614B & 3250 & M4 & $4.5^{b}$ & & & & & 4.4 & \\
\hline J154433.9-335254 & 3050 & M5.5 & 4.0 & 1.7 & -1.55 & 0.11 & 4.5 & 32.1 & -1.8 \\
\hline \multicolumn{10}{|l|}{ Lupus 3} \\
\hline J160528.6-384621 & 3000 & M6 & 4.0 & 1.0 & -1.27 & 0.09 & 2.0 & 22.3 & - \\
\hline J160755.2-390603 & 2950 & M6.5 & 3.5 & 2.3 & -1.32 & 0.09 & 1.0 & 23.0 & -2.6 \\
\hline J160804.8-390449 & 2900 & M7 & 3.5 & 2.7 & -1.58 & 0.06 & 1.2 & 16.4 & -2.1 \\
\hline J160833.0-385222 & 2800 & M7.5 & 3.5 & 0.8 & -1.42 & 0.06 & 1.0 & 20.3 & -2.7 \\
\hline J160835.5-390035 & 2950 & M6.5 & 3.5 & 0.5 & -0.92 & 0.10 & 1.3 & 12.8 & -2.5 \\
\hline J160836.2-392302 & 4400 & K5 & 4.0 & 6.3 & 0.75 & & $<1$ & 12.4 & -1.3 \\
\hline J160839.8-392922 & 3100 & M5 & 3.5 & 1.0 & -0.83 & 0.20 & 1.0 & 8.9 & - \\
\hline J160855.8-382633 & 3050 & M5.5 & 4.0 & 1.1 & -1.32 & 0.13 & 3.6 & 10.1 & - \\
\hline J160904.6-392112 & 3100 & M5 & 3.5 & 1.6 & -0.32 & & $<1$ & 6.4 & -2.8 \\
\hline J160915.7-385139 & 3100 & M5 & 3.5 & 0.0 & -1.09 & 0.13 & 1.8 & 4.1 & -3.1 \\
\hline J160920.8-384510 & 3000 & M6 & $5.0^{b}$ & & & & & 6.2 & - \\
\hline $\mathrm{J} 160922.8-385550 \mathrm{~A}$ & 3100 & M5 & 3.5 & 1.0 & -0.46 & & $<1$ & 9.1 & -2.8 \\
\hline J160922.8-385550B & 3250 & M4 & 3.5 & & & & & 5.2 & -2.8 \\
\hline J160928.0-384853 & 3050 & M5.5 & 3.5 & 1.6 & -0.86 & 0.20 & 1.0 & 9.8 & - \\
\hline J161013.9-375958 & 3000 & M6 & 3.5 & 0.8 & -1.10 & 0.11 & 1.0 & 49.3 & -2.8 \\
\hline J161030.6-383151 & 3000 & M6 & 4.0 & 0.5 & -1.56 & 0.08 & 3.6 & 9.1 & -2.8 \\
\hline J161033.2-383023 & 3200 & M4.5 & 3.5 & 1.5 & -0.31 & & $<1$ & 7.7 & -2.7 \\
\hline J161041.9-382304 & 3100 & M5 & 3.5 & 0.1 & -0.61 & 0.20 & 1.0 & 10.5 & -2.9 \\
\hline J161059.0-391451 & 3100 & M5 & 4.0 & 0.5 & -1.07 & 0.20 & 2.0 & 7.6 & -2.7 \\
\hline J161138.1-384135 & 3500 & M2.5 & $5.0^{b}$ & & & & & 4.1 & -2.9 \\
\hline J161207.6-381324 & 3200 & M4.5 & 3.5 & 1.1 & -0.48 & 0.30 & 1.0 & 3.6 & -2.8 \\
\hline J161210.4-390904 & 2950 & M6.5 & 4.0 & 1.3 & -1.72 & 0.06 & 3.2 & 14.0 & $-2.5^{c}$ \\
\hline J161225.6-381742 & 3000 & M6 & 3.5 & 0.1 & -1.59 & 0.11 & 4.0 & 21.3 & $-2.5^{c}$ \\
\hline \multicolumn{10}{|l|}{ Lupus 4} \\
\hline J160019.2-415635 & 3050 & M5.5 & $5.0^{b}$ & & & & & 5.5 & -2.7 \\
\hline J160359.6-421356 & 3000 & M6 & 3.5 & 0.9 & -1.97 & 0.08 & 7.2 & 13.7 & - \\
\hline J160348.1-414044 & 2950 & M6.5 & 3.5 & 0.1 & -1.54 & 0.07 & 1.0 & 16.2 & - \\
\hline
\end{tabular}

Notes. ${ }^{(a)}$ Quantities estimated assuming a distance of $150 \mathrm{pc}$ (Lupus 1 and 4) or $200 \mathrm{pc}$ (Lupus 3). These estimates are invalid if the objects are in the foreground, as suspected for those with $\log g>4 .{ }^{(b)}$ Possibly non-member on the basis of its high $\log g$ values. ${ }^{(c)}$ Spectral index computed between $2.2 \mu \mathrm{m}$ and $8 \mu \mathrm{m}$.
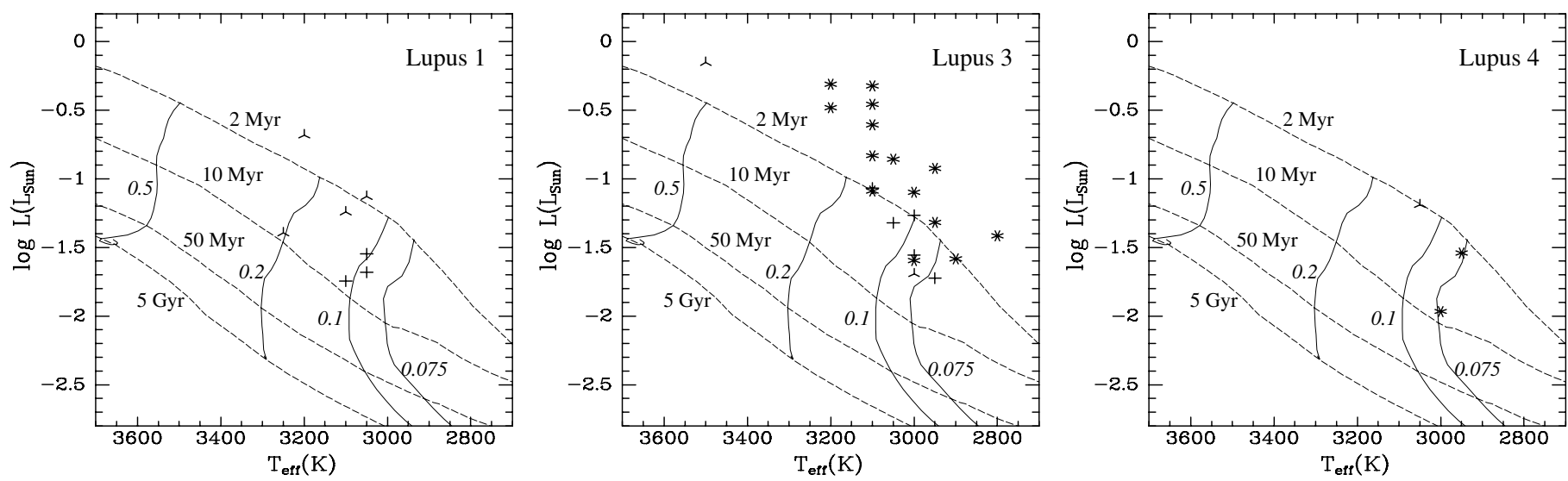

Fig. 5. Temperature-luminosity plots of the nongiant stars observed in Lupus 1, 3, and 4. Superimposed are selected evolutionary tracks of Baraffe et al. (1998) for stars above the substellar limit, merged with those of Chabrier et al. (2000) for brown dwarfs with dusty atmospheres. Objects with estimated $\log g \simeq 3.5$ are marked as asterisks; those with $\log g \simeq 4.0$, with crosses; and those with $\log g \simeq 4.5-5.0$, with three-pointed stars. 


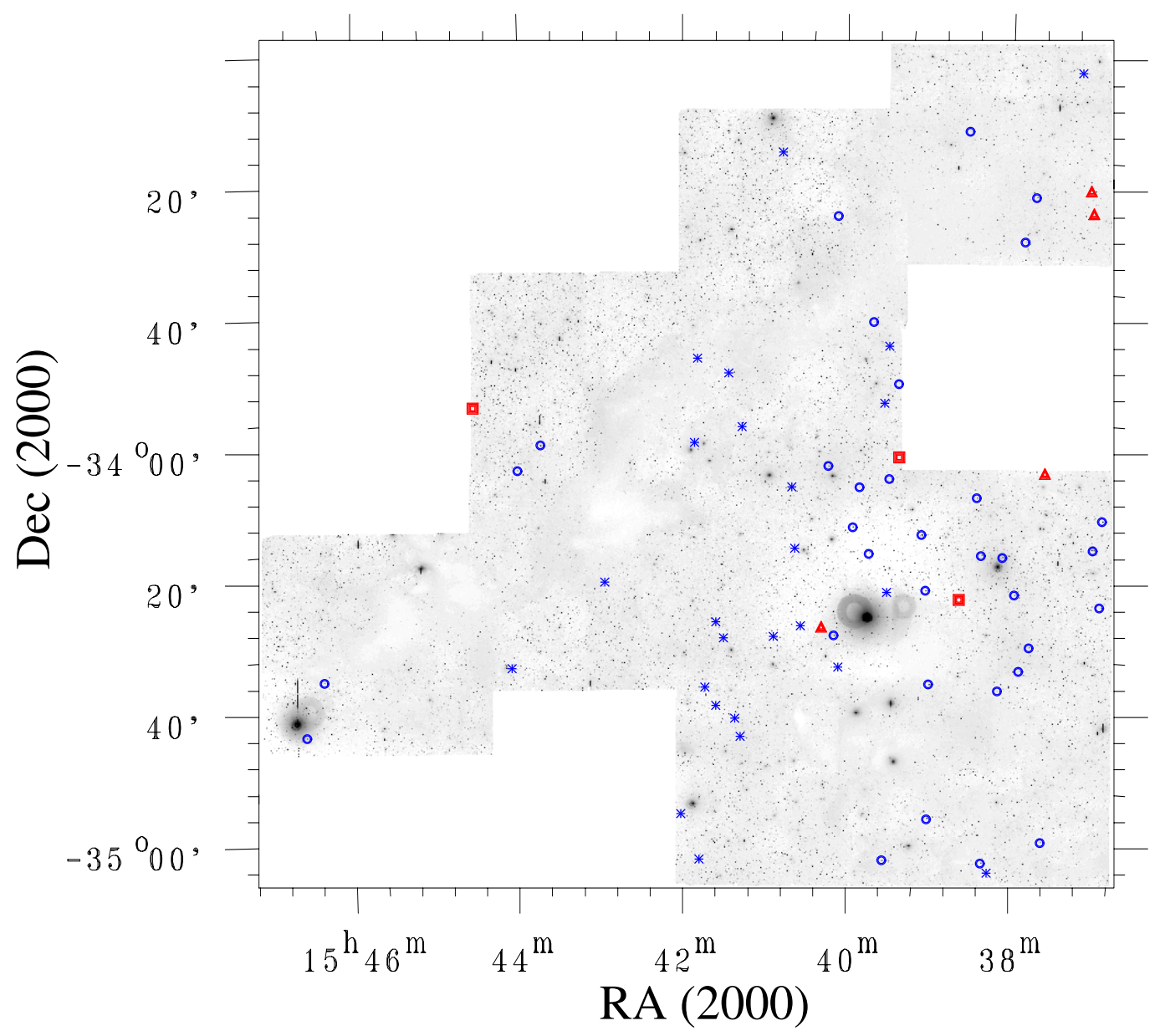

Fig. 6. Position of the new Lupus 1 candidate members proposed by CSL, superimposed on a $R$-band image of the region from that work. Red squares mark the position of nongiants spectroscopically confirmed as members by the observations presented here. Red triangles are also nongiants, but having $\log g \gtrsim 4.5$ according to the depth of the NaI feature near $8190 \AA$ A. Blue circles mark the position of spectroscopically confirmed giants, and therefore non-members. Blue asterisks are candidate members identified by CSL, but for which no spectroscopy is available yet.

position in the $T_{\text {eff }}-L$ diagram, which rather shows hints of the reverse trend. This suggests that the objects with $\log g \gtrsim 4.5$ are located at a closer distance from the Sun than those with lower values of $\log g$.

No particular concentration of these objects is seen in their positions on the sky, presented in Fig. 6. As the figure shows nearly half of the candidates photometrically selected by CSL are still awaiting spectroscopic confirmation, but in view of the success rate obtained from the objects for which spectroscopy is now available we expect the number of additional nongiants to be only a few. It is thus very unlikely that the results discussed here on Lupus 1 will change significantly once the entire sample has been observed.

\subsection{Lupus 3}

The situation is different in Lupus 3, where the fraction of nongiants among our objects is almost 50\%. The existence of the distributed population is clearly confirmed, and the $\log g$ values of its members indicate in general very young ages, with most of them being best fitted by $\log g \simeq 3.5$. In terms of NaI strength only two objects, J160920.8-384510 and J161138.1-384135, clearly stand out from the rest, having depths comparable to those of field stars. Objects with $\log g \simeq 4.0$ tend to be located below those with lower surface gravities in the $T_{\text {eff }}-L$ diagram, as expected if both groups are located at the same distance. Nevertheless, this trend is not shared by the two objects with higher surface gravities, most notably by the M2.5 star 161138.1-384135. The band populated by nongiants in Lupus 3 is clearly offset with respect to that defined by the same objects in Lupus 1, as can be seen by comparing the first two panels in Fig. 5. Part of this offset may be due to the adopted distances to each cloud, as we have assumed Lupus 3 to lie $50 \mathrm{pc}$ farther than Lupus 1 in accordance with the discussion in Comerón (2008). The greater distance to Lupus 3 is based on the HiPPARcos trigonometric parallax for the bright Herbig Ae/Be pair HR 5999/HR 6000, located near the center of the main cloud of Lupus 3, whereas the extinction jump method for distance determination applied to Lupus 1 (Lombardi et al. 2008) strongly favors a distance of $150 \mathrm{pc}$ for that cloud. If a common distance of $150 \mathrm{pc}$ were adopted for both Lupus 1 and Lupus 3, the position of the data points in the $T_{\text {eff }}-L$ diagram corresponding to Lupus 3 would be shifted downward by only 0.25 dex in luminosity, giving a somewhat better overlap between the positions of the $\log g=4.0$ stars in the $T_{\text {eff }}-L$ diagram of both clouds but otherwise leaving our results qualitatively unchanged. Although the derived ages in Lupus 3 would therefore change, the increase would be fairly limited due to the fast evolution in luminosity in the early stages of pre-main sequence contraction, and the revised ages would still be in good agreement with the values of $\log g$ estimated from the spectra. 


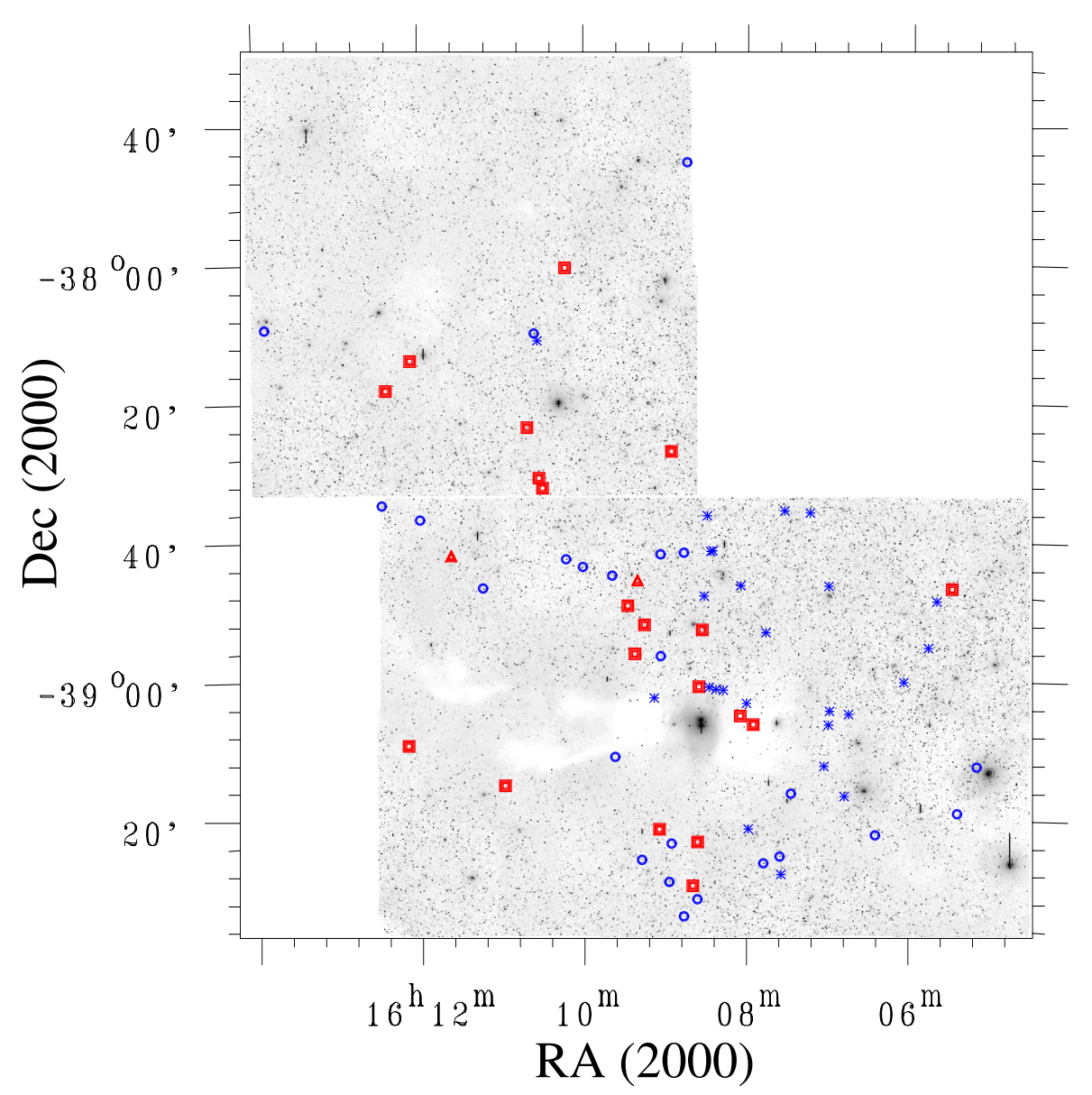

Fig. 7. Same as Fig. 6 for the Lupus 3 region.

The population around Lupus 3 is rich in later spectral types, reflecting a well-known trend already noted by Hughes et al. (1994) in the population spatially projected on the main cloud. The two objects with the latest spectral types, J160804.8-390449 (M7) and J160833.0-385222 (M7.5), are most likely substellar, and the eight additional M6-M6.5 objects are close the substellar limit. Both the overall distribution of our spectral types and the position of our objects in the $T_{\text {eff }}-L$ diagram are very similar to those of the much larger, mostly "on-cloud" complementary sample spectroscopically observed by Mortier et al. $(2011)^{2}$. While this suggests that the intrinsic properties of both stellar populations are very similar in terms of underlying mass distribution and age, it must be noted that the sample of Mortier et al. (2011) is largely composed of young stellar objects identified through strong $\mathrm{H} \alpha$ emission and infrared excess caused by circumstellar material. By contrast, most of the objects of our sample lack any substantial infrared excesses, as noted by CSL, and the $\mathrm{H} \alpha$ emission that we measure is in general weak to moderate. Only the star with the largest $\mathrm{H} \alpha$ equivalent width, J161013.9-375958, displays simultaneous optically thick CaII triplet emission, which is normally attributed to accretion (Herczeg \& Hillenbrand 2008).

Also in Lupus 3 we have discovered a binary system due to the chance alignment of the slit with the position angle of the

\footnotetext{
2 Mortier et al.'s (2011) study includes sources in the same regions that we study in the present work. However, more than $85 \%$ of their sample is composed of Lupus 3 members and it includes a single member of Lupus 1 . Therefore, the collective properties studied in that work reflect essentially those of the Lupus 3 population.
}

pair. The J160922.8-385550 system is formed by two components with M4 and M5 types respectively, both in the $\log g \simeq$ 3.5 group and with weak $\mathrm{H} \alpha$ emission.

Figure 7 shows that the spectroscopically confirmed young stars are weakly concentrated toward the main cloud of Lupus 3. The region around this cloud contains 25 additional candidates still to be spectroscopically observed, which may add over ten more members if the ratio between giants and nongiants among these objects remains similar to that found among the spectroscopically observed sample.

\subsection{Lupus 4}

Although the number of candidates identified by CSL in Lupus 4 is small, also in this region we find both giants and nongiants represented. The two giants, J155921.8-412808 and J160336.9-414209, have respectively early-M and late-M spectral types and their spectra are shown in Fig. 1. Of the three remaining objects, one is a M5.5 star of high surface gravity and weak $\mathrm{H} \alpha$ emission, whereas the other two have M6 and M6.5 types respectively, $\log g \simeq 3.5$, and stronger, but still moderate, $\mathrm{H} \alpha$ emission. Their spectra, shown in Fig. 4, clearly shows the difference in the strength of the NaI feature. All the objects appear well detached from the Lupus 4 molecular cloud.

Like in the other clouds, the object with the highest value of $\log g$ does not lie below those with lower values in the $T_{\text {eff }}-L$ diagram produced assuming the same distance to all three objects, but rather the opposite. We thus consider its membership in the Lupus 4 star forming region unlikely. The location in 


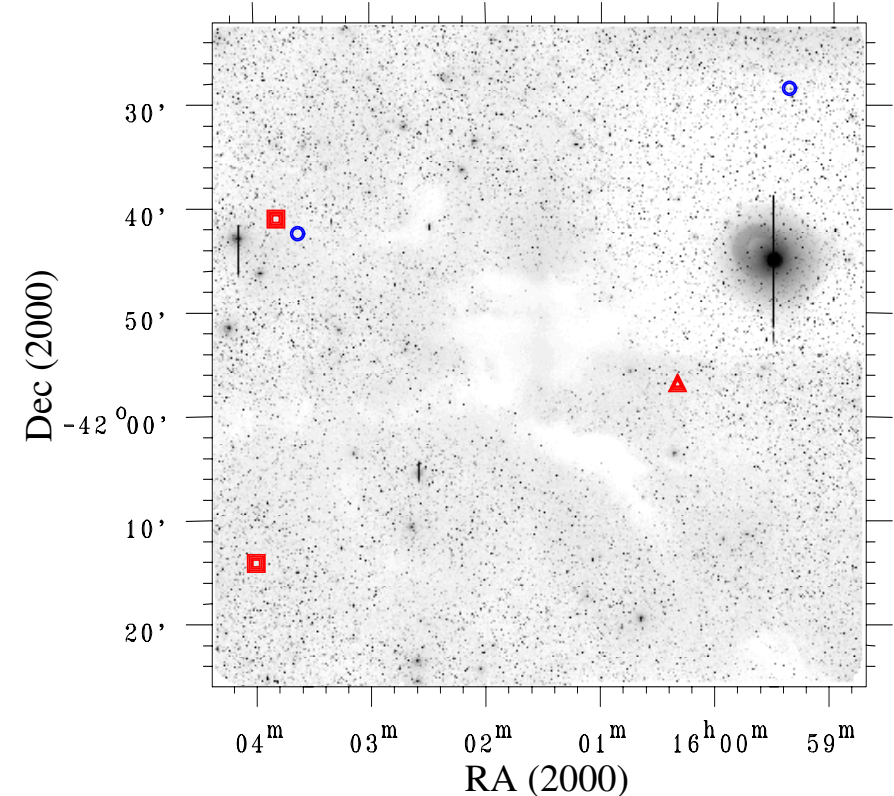

Fig. 8. Same as Fig. 6 for the Lupus 4 region.

the diagram of the other two objects, whose masses are near or slightly below the substellar limit, is consistent with the young ages indicated by their low surface gravities, although the age of 9 Myr estimated for J160359.6-421356 from the evolutionary tracks appears too large for its low surface gravity.

\subsection{The dispersed population of Lupus 3}

The dispersed component of Lupus 3 is largely formed by a population of young stars and brown dwarfs similar to that of the previously known objects residing in the cloud, consistent with their inferred surface gravities and with a common distance. Their mass distributions also appear to be similar, as suggested by the large abundance of M5 and later stars and brown dwarfs as compared to earlier spectral types both in the on-cloud and the dispersed populations. We note however that the cutoff at $T_{\text {eff }} \simeq 3400 \mathrm{~K}$ in the photometric selection by CSL limits the sampling of the mass range to cool objects, and that possible higher-mass objects that may exist among this population are thus excluded from their sample. The corresponding association of the confirmed nongiants in this $\log g$ range with the Lupus 1 and 4 clouds is also likely. In Lupus 1 the dispersed population seems to be older than in Lupus 3, as hinted by the lack of objects with $\log g \simeq 3.5$ in our sample, although the small number of such objects in those other clouds prevents us from drawing any firm conclusions on this.

To study the dispersed population of Lupus 3 while avoiding biases that may be introduced by the way in which members are selected, we use a sample composed of our newly classified members excluding those with $\log g=4.5-5.0$, to which we add the already known members of Lupus 3 that are also selected by the CSL criterion of identification of candidates. The intrinsic properties (including mass and age) of the known members have been derived in exactly the same way as for the dispersed population, and their mid-infrared spectral indices $n$ have been taken from Merín et al. (2008). We have then split this sample into an "on-cloud" population, composed by 29 objects whose positions lie within a circle that contains the

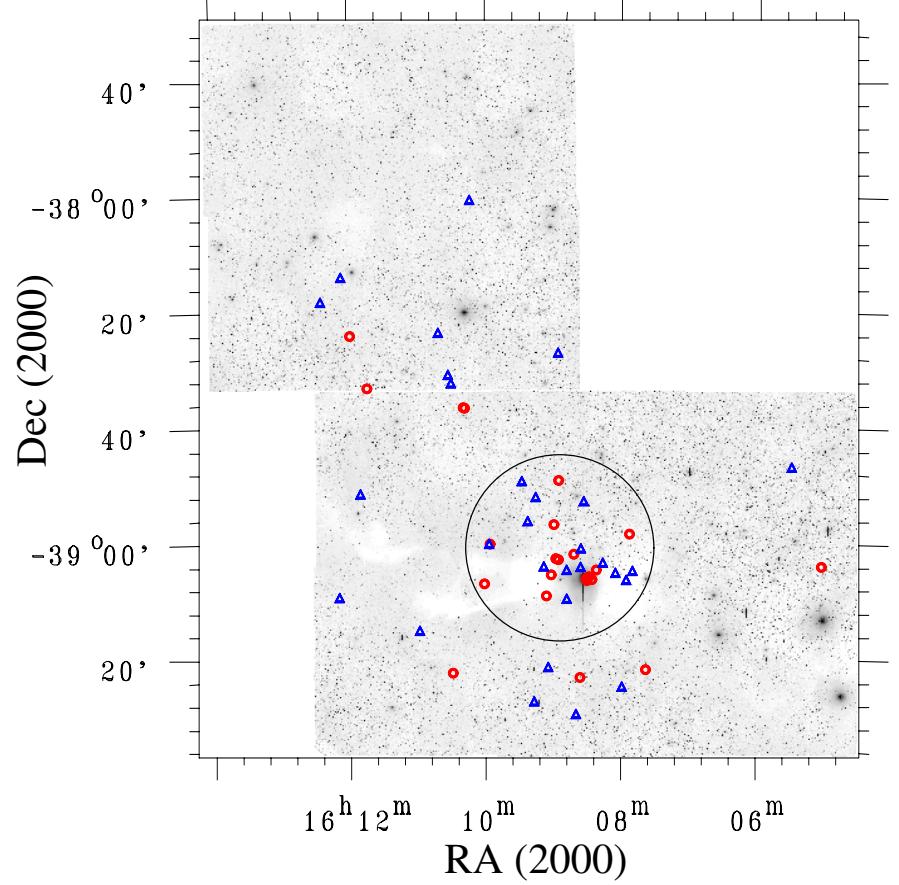

Fig. 9. $R$-band image of Lupus 3 (from CSL) marking the positions of spectroscopically confirmed members having an estimated $\log g=$ 3.5-4.0 that fulfill the criterion described by CSL to identify possible members of the Lupus clouds, including those members already identified in previous studies. Red circles represent objects with significant infrared excess (spectral index $n>-2$ ) and blue triangles are objects with moderate or no excess. The large circumference marks the limits of the area chosen to split the sample into an "on-cloud" population and a "distributed" population, as discussed in the text.

main molecular concentration in Lupus 3, and a "distributed" population of 24 objects outside this circle, in the way shown in Fig. 9. New members confirmed with our observations can be found in both regions.

A Kolmogorov-Smirnov test of the distribution of derived ages of both populations shows that they are different only at a rather modest $90 \%$ confidence level, leading us to consider any age difference between them as marginal at most. The difference between the age distributions of stars with and without important infrared excess (defining the boundary between both sets at $n=-2)$ is even more marginal.

A more significant difference is found in the abundance of circumstellar material, revealed by spectral indices significantly above $n=-2$. As Fig. 9 shows, stars with infrared excess are present in both the on-cloud and the distributed population, and Fig. 10 shows the spectral indices as a function of the stellar age for each population. A split between sources with clear infrared excess $(n>-2)$ and those with weak or no excess $(n<-2$, including in this category sources undetected by Spitzer), shows that 15 of the 29 on-cloud sources $(52 \% \pm 9 \%$ with $1-\sigma$ uncertainty) have $n>-2$, whereas the fraction decreases to 8 out of 24 among the distributed population $(33 \% \pm 10 \%)$.

Distributed populations of low-mass stars are known around other star-forming regions, such as Taurus (Feigelson 1996, Findeisen \& Hillebrandt 2010), Chamaeleon (Feigelson 1996; Luhman 2007), Orion (Biazzo et al. 2012), $\rho$ Ophiuchi (Wilking et al. 2005), R Coronae Australis (Neuhäuser et al. 2000) or the Rosette nebula (Wang et al. 2009). It has been suggested that such populations may be due to the drift of stars away from their 


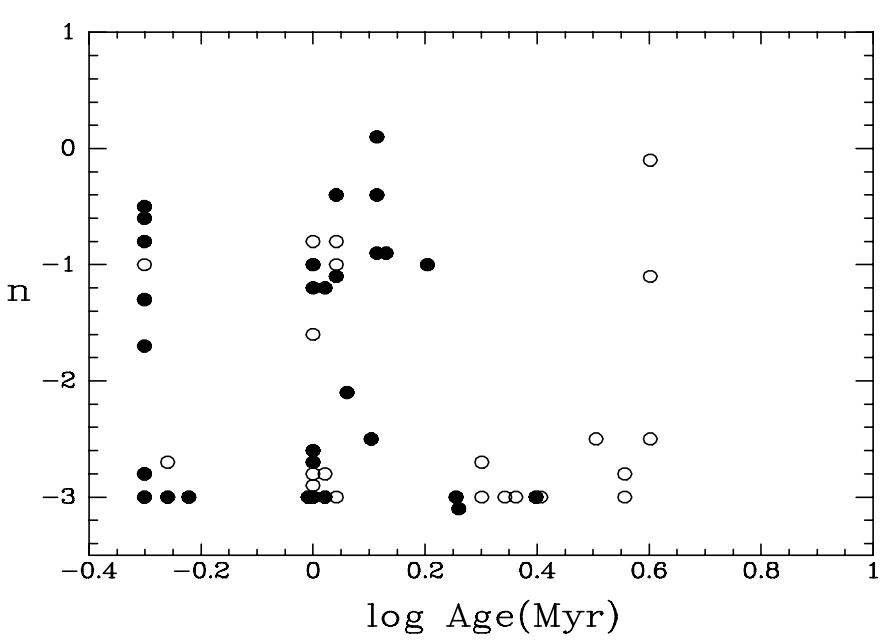

Fig. 10. Infrared excess (measured by the spectral index $n$ ) as a function of age for all spectroscopically confirmed members having $\log g=$ 3.5-4.0 that fulfill the criterion described by CSL to identify possible members of the Lupus clouds. The sample includes those objects already identified in previous works. Filled circles represent objects in the "on-cloud" population as defined in the text (see also Fig. 9), and open circles are members of the dispersed population.

formation sites (Feigelson 1996), that they have formed in-situ in short-lived clouds formed by converging flows of atomic gas (Ballesteros-Paredes et al. 1999), or that they may be the result of the ejection from star-forming sites by gravitational interaction with other members (Armitage \& Clarke 1997; Reipurth et al. 2010). The interpretation of extended populations may be complicated by the presence of additional components not directly related to them, such as the Gould Belt population that is seen in the direction of Lupus (Wichmann et al. 1997) or Orion (Biazzo et al. 2012).

The population with little or no infrared excess and low levels of $\mathrm{H} \alpha$ emission can be identified as the low-mass component of the weak T-Tauri star (WTTS) populations of star forming regions generally revealed by their X-ray emission (Krautter et al. 1997). Evidences for an age of this population generally older than that of classical T Tauri stars (CTTS) in the same star forming regions have been provided by Cieza et al. (2007) and Bertout et al. (2007). These studies show that CTTS tend to be more concentrated toward clouds than WTTS and that WTTS tend to be older, although some young stars seem to lose their disks very early into their evolution and display WTTS characteristics already at a very early age. Interestingly, as noted above our data provide no evidence for a significant difference in the age distributions between the CTTS and the WTTS populations, thus suggesting that there is no noticeable transition from the CTTS to the WTTS phases during the age span covered by our sample.

If confirmed, the increase in the fraction of WTTS with increasing distance to the main star forming site that we identify in our sample, coupled with the lack of significant differences in the distributions of ages between the samples with and without strong infrared excess, or between the on-cloud and dispersed samples, would have interesting implications on the origin of the distributed population. If the members of the oncloud and dispersed population formed over the same time span in the main Lupus 3 dark cloud, the dispersed population may simply represent the high end of the initial velocity distribution. However, in that case we would not expect to find any significant differences in the distributions of infrared excesses between the on-cloud and the distributed populations, contrarily to what our results hint. The same is true if the distributed population formed in-situ in short-lived clouds simultaneously with the on-cloud population, unless the process leading to the formation of the distributed population tended to favor short-lived disks. This is a possibility that was already discussed by CSL, who suggested that the process of externally triggered star formation causing an outside-in collapse of preexisting clouds might leave its signature in the structure of circumstellar envelopes and disks.

An additional possibility is that the distributed population may be composed primarily of objects formed in the main cloud and subsequently ejected through close gravitational interactions with small stellar aggregates. Armitage \& Clarke (1997) have shown that such interactions result in the truncation and fast evolution of the circumstellar disks of the ejected objects. Observations of infrared excesses among binary members of young star forming reasons have found a decrease in their frequency for short separations between components (Cieza et al. 2009), in agreement with theoretical expectations and testifying to the importance of short-range dynamical interactions in the disruption of disks. The expected ejection velocities, of a few $\mathrm{km} \mathrm{s}^{-1}$, would be consistent with the presence in our sample of objects having projected distances of over $3 \mathrm{pc}$ to the star forming cloud and estimated ages around $1 \mathrm{Myr}$, implying tangential velocities in excess of $\sim 3 \mathrm{~km} \mathrm{~s}^{-1}$. The distributed population would thus have an age distribution similar to that of the oncloud population but a smaller fraction of circumstellar disks, as hinted by our observations. A similar mechanism has been invoked by Reipurth et al. (2010) to explain the existence of distant companions to protostars as ejected members from triple systems, which ultimately escape from the potential well of the star forming cloud. Binaries would be expected to be rare among ejected objects, although not completely absent as the recoil of the remaining bound binary following the ejection of a member of an initially triple system would result in the ejection of the binary as well. The hypothesis of ejection to account for the origin of the dispersed population in Lupus 1 and 3 was considered to be unlikely by CSL on the grounds of the apparently high abundance of members of the distributed population, which required a very high efficiency of the ejection mechanism. However, the new results presented here that reduce the membership of the dispersed population, most notably in Lupus 1, renders the ejection hypothesis more viable. If ejections are relatively common in dense star forming aggregates where dynamical interactions abound, as our results on Lupus 3 hint, the subsequent frequent disruption of disks may result in a lower fraction of planetary systems around stars formed in such environments.

\subsection{The older population}

The positions in the $T_{\text {eff }}-L$ diagrams of the seven objects with surface gravity near or above $\log g \simeq 4.5$ in our sample with respect to younger, lower surface gravity objects suggest that the former are foreground to the clouds, thus appearing in the band occupied by young members of the clouds by virtue of their closer distance despite their lower intrinsic luminosities. They may be field stars of the general galactic disk population spanning a wide range of ages and distances to the Sun, but they may also be low-mass members of the weak $\mathrm{T}$ Tauri stars population in the direction of Lupus first reported by Krautter et al. (1997) based on ROSAT X-ray observations. Wichmann et al. (1997) associated the weak T Tauri stellar 
population with the Gould Belt on the basis of the positional coincidence on the sky, and suggested that they may lie at the same distance as the Lupus clouds. However, the assumption of a similar distance by Wichmann et al. (1997) appears to be based on the idea that the Gould Belt is a ring whose stars lie all at the same distance in a given direction, rather than on an independent estimate of the distance of the stars. Later on, Guillout et al. (1998) showed that the weak T Tauri stellar component traces the Gould Belt at all galactic longitudes and found evidence that the members of this population are distributed in a disk, rather than a ring. More recently, Biazzo et al. (2012) have used follow-up observations of the weak T Tauri star population revealed by ROSAT in the direction of Orion to produce similar results, identifying three components respectively associated with the young population of the Orion giant molecular clouds, the Gould Belt, and the evolved population of the galactic disk. Interestingly, two aggregates of X-ray emitting stars associated with the second component are found at an estimated distance of $140 \mathrm{pc}$, much closer to the Sun than the Orion complex and with ages in the ranges $2-10 \mathrm{Myr}$ and 2-20 Myr, suggesting that also in the direction of Orion the Gould Belt spans a wide range of distances and ages. Therefore, our conclusion that these objects are in the foreground of the Lupus clouds does not rule out their association with the Gould Belt.

The $\mathrm{H} \alpha$ emission that almost all these objects display is very common among field $\mathrm{M}$ stars of late spectral types, most of which are classified as $\mathrm{dMe}$ as already shown by Joy \& Abt (1974) and confirmed with the large sample drawn from the Sloan Digital Sky Survey (West et al. 2004). Also, the low $\mathrm{H} \alpha$ equivalent widths are in all cases similar to those measured among dMe stars (Riaz et al. 2006). The pervasiveness of $\mathrm{H} \alpha$ emission among the objects with high surface gravity thus cannot be taken in principle as an indication of youth, as the magnetic activity that causes the $\mathrm{H} \alpha$ emission does not decay with time in fully convective stars (Chabrier \& Küker 2006). The only exception may be 161138.1-384135, a M2.5 star in the direction of Lupus 3. Early $\mathrm{M}$ dwarfs are not fully convective, and their magnetic field is generated by a dynamo mechanism coupled with rotation that, unlike in fully convective objects, does decay with time as angular momentum decreases through magnetic breaking. Indeed, West et al. (2004) find that only about 5\% of all M2.5 stars display $\mathrm{H} \alpha$ in emission, as opposed to $80 \%$ at M8. Thus, on statistical grounds $161138.1-384135$ is likely to be a moderately young star located in front of Lupus 3. The remaining stars were selected as candidate members because of the expected lack of field stars with the combination of temperature and apparent magnitude in the direction of Lupus (see CSL). Their membership in the Gould Belt would thus explain the abundance of these objects with respect to the expectation if only the field population is taken into account. Their surface gravities are consistent with age estimates of the Gould Belt, 30-60 Myr, as discussed by Comerón (1999).

\section{Conclusions}

The new observations presented here provide spectroscopic confirmation of the new population of low-mass young stars in and around the Lupus clouds reported by Comerón et al. (2009; CSL), while modifying some conclusions of that work. Our findings can be summarized as follows:

- As many as $65 \%$ of the possible members identified by CSL, particularly in Lupus 1, are shown to be giants, mostly of
$\mathrm{K}$ type. However, we are able to spectroscopically confirm 26 candidates in Lupus 1, 3, and 4 as nongiant, late-type objects that display low-surface gravity features typical of young stellar objects. In addition, we identify 8 suspected late-type field stars.

- Based on the surface gravity-sensitive NaI feature at $8190 \AA$ we confirm that the vast majority of these objects, particularly in Lupus 3, are very young very low-mass stars or massive brown dwarfs, with spectral types comprised between M4 and M7.5 and consistent with them being located at the same distance as the previously known population of the various Lupus clouds.

- All the confirmed candidates have moderate $\mathrm{H} \alpha$ emission. Their fluxes in the $3.6 \mu \mathrm{m}-24 \mu \mathrm{m}$ generally indicate little or no excess emission by warm circumstellar material.

- Objects with estimated surface gravities $\log g \simeq 4.0$ tend to be less luminous than those having $\log g \simeq 3.5$, as expected from evolutionary effects. This trend is not followed by objects with estimated $\log g>4.0$, which we consider to be most likely unrelated to the Lupus forming clouds.

- The age distribution of the new confirmed members of Lupus 3, the only region where the census is rich enough to carry out statistical comparisons, does not differ significantly from that of previously known lightly obscured members.

- When splitting the samples of lightly obscured Lupus 3 members between an "on-cloud" population near the main concentration of molecular gas and dust and a dispersed population, again no significant differences are detected in the age distributions of their respective members. However, the dispersed population seems to have a lower fraction of objects with infrared excess caused by warm circumstellar material.

- The similar age distribution of the "on-cloud" and the dispersed populations, combined with the decrease in the fraction of objects with substantial infrared excesses among the latter, leads us to consider dynamical ejection from the main cloud as a viable mechanism to account for the properties of the dispersed population.

- We favor an origin in the foreground part of the Gould Belt for the few newly discovered objects with higher surface gravities.

The results presented here provide useful new material to understand the relationship between the star forming regions in Lupus, most notably Lupus 3, and their surroundings. The newly identified members confirm the existence of a population of objects whose signatures of youth are weak, thus rendering them difficult to identify by surveys based on them.

Many of these members belong to the dispersed population of the clouds, whose origin is not yet completely understood. New observations could however yield new light and either confirm or rule out possible explanations. In the first place, many of the candidate members identified in our previous work (Comerón et al. 2009) still await spectroscopic confirmation, which once obtained could increase the significance of statistical trends hinted by the results presented here. On the other hand, binarity is a barely explored aspect of the extended population that may contain decisive information on its origin, given the predictions of ejection models of a reduction in the numbers of binaries. Finally, highly accurate kinematics coming from Gaia astrometry combined with radial velocities measured from the ground could allow us to determine the trajectories of these objects and trace them back to their birth places. 
Acknowledgements. We are pleased to thank the staff of the Paranal Observatory for the execution of our observations in Service Mode, as well as to Dr. Mario van den Ancker, of the User Support Department of the ESO Data Management and Operations Division, for his support in the preparation of the Phase 2 for our observations. We also thank Dr. Bo Reipurth for inspiring discussions on the possible origin of the extended population studied in this paper, and the anonymous referee for a detailed report that helped improve the presentation of our results. F.C. wishes to express his gratitude to the Centro de Astrobiología (INTA-CSIC) and the European Space Astronomy Centre of the European Space Agency near Madrid, Spain, as well as to the staff of the Specola Vaticana, for their hospitality at various stages of this work. B.L.M. thanks financial support by the Spanish former Ministry of Science and Innovation (MICINN) through grant Consolider-CSD2006-00070. This research has made use of data products from the Two Micron All Sky Survey, which is a joint project of the University of Massachusetts and the Infrared Processing and Analysis Center/California Institute of Technology, funded by the National Aeronautics and Space Administration and the National Science Foundation.

\section{References}

Allard, F., Hauschildt, P. H., \& Schwenke, D. 2000, ApJ, 540, 1005 Armitage, P. J., \& Clarke, C. J. 1997, MNRAS, 285, 540

Ballesteros-Paredes, J., Hartmann, L., \& Vázquez-Semadeni, E. 1999, ApJ, 527, 285

Baraffe, I., Chabrier, G., Allard, F., \& Hauschildt, P. H. 1998, A\&A, 337, 403

Bertout, C., Siess, L., \& Cabrit, S. 2007, A\&A, 473, L21

Biazzo, K., Alcalá, J. M., Covino, E., et al. 2012, A\&A, 542, A115

Chabrier, G., \& Küker, M. 2006, A\&A, 446, 1027

Chabrier, G., Baraffe, I., Allard, F., \& Hauschildt, P. 2000, ApJ, 542, 464

Cieza, L., Padgett, D. L., Stapelfeldt, K. R., et al. 2007, ApJ, 667, 308

Cieza, L., Padgett, D. L., Allen, L. E., et al. 2009, ApJ, 696, L84

Comerón, F. 1999, A\&A, 351, 506

Comerón, F. 2008, in Handbook of Star Forming regions, ed. B. Reipurth, ASP

Monographs, 2

Comerón, F., Spezzi, L., \& López Martí, B. 2009, A\&A, 500, 1045 (CSL)
Decin, L., Vandenbussche, B., Waelkens, C., et al. 2003, A\&A, 400, 709 Evans, N. J. II, Allen, L. E., Blake, G. A., et al. 2003, PASP, 115, 965

Evans, N. J. II, Dunham, M. M., Jørgensen, J. K., et al. 2009, ApJS, 181, 321

Feigelson, E. D. 1996, ApJ, 468, 306

Feigelson, E. D., \& Montmerle, T. 1999, ARA\&A, 37, 363

Findeisen, K., \& Hillenbrand, L. 2010, AJ, 139, 1338

Fluks, M. A., Plez, B., The, P. S., et al. 1994, A\&AS 105, 311

Gorlova, N. I., Meyer, M. R., Rieke, G. H., \& Liebert, J. 2003, ApJ, 593, 1074

Guillout, P., Sterzik, M. F., Schmitt, J. H. M. M., Motch, C., \& Neuhäuser, R. 1998, A\&A, 337, 113

Herczeg, G. J., \& Hillenbrand, L. A. 2008, ApJ, 681, 594

Hughes, J. H., Hartigan, P., Krautter, J., \& Kelemen, J. 1994, AJ, 108, 1071

Joy, A. H., \& Abt, H. A. 1974, ApJS, 28, 1

Kirkpatrick, J. D., Henry, T. J., \& McCarthy, D. W. 1991, ApJS, 77, 417

Krautter, J., Wichmann, R., Schmitt, J. H. M. M., et al. 1997, A\&AS, 123, 329

Lombardi, M., Lada, C. J., \& Alves, J. 2008, A\&A, 480, 785

López Martí, B., Jiménez-Esteban, F., \& Solano, E. 2011, A\&A, 529, A108

Luhman, K. L. 2007, ApJS, 173, 104

Luhman, K. L., Allen, P. R., Espaillat, C., Hartmann, L., \& Calvet, N. 2010, ApJS, 186, 111

Merín, B., Jørgensen, J., Spezzi, L., et al. 2008, ApJS, 177, 551

Mortier, A., Oliveira, I., \& van Dishoeck, E. F. 2011, MNRAS, 418, 1194

Neuhäuser, R., \& Comerón, F. 1999, A\&A, 350, 612

Neuhäuser, R., Walter, F. M., Covino, E., et al. 2000, A\&AS, 146, 323

Ray, T. P. 2007, in Jets from Young Stars, Lecture Notes in Physics (Berlin: Springer-Verlag), 723

Reipurth, B., Mikkola, S., Connelley, M., \& Valtonen, M. 2010, ApJ, 725, L56

Riaz, B., Mullan, D. J., \& Gizis, J. E. 2006, ApJ, 650, 1133

Skrutskie, M. F., Cutri, R. M., Stiening, R., et al. 2006, AJ, 131, 1163

Spezzi, L., Alcalá, J. M., Frasca, A., et al. 2007, A\&A, 470, 281

Wang, J., Feigelson, E. D., Townsley, L. K., et al. 2009, ApJ, 696, 47

West, A. A., Hawley, S. L., Walkowicz, L. M., et al. 2004, AJ, 128, 426

Wichmann, R., Sterzik, M., Krautter, J., Metanomski, A., \& Voges, W. 1997, A\&A, 326, 211

Wilking, B. A., Meyer, M. R., Robinson, J. G., \& Greene, T. P. 2005, AJ, 130, 1733 